\title{
Stability of Grain Nutrient Concentrations in White Finger Millet
}

\author{
H.S. Saritha ${ }^{1^{*}}$, P. Ravishankar ${ }^{2}$ and N.C. Sunitha ${ }^{1}$ \\ ${ }^{1}$ Department of Genetics and Plant Breeding, UAS, Bengaluru, Karnataka, India \\ ${ }^{2}$ AICRP on Small millets, ZARS, UAS - Bengaluru, Karnataka, India \\ *Corresponding author
}

\begin{tabular}{|l|}
\hline Ke y w o r d s \\
$\begin{array}{l}\text { Finger millet, Genotype } \times \\
\text { environment, Stability } \\
\text { parameters }\end{array}$ \\
\hline Article Info \\
\hline $\begin{array}{l}\text { Accepted: } \\
22 \text { October } 2018 \\
\text { Available Online: } \\
10 \text { November } 2018\end{array}$ \\
\hline
\end{tabular}

\section{Introduction}

Finger millet (Eleusine coracana $\mathrm{L}$. Gaertn.) sub species coracana belongs to family Poaceae. Eleusine is a generic term named

after the Greek goddess of the cereals.

\section{A B S T R A C T}

Grain calcium, iron and zinc are important micronutrients for human health for which deficiency occurs widespread in the world. Knowledge on genotype $\times$ environment interaction of these nutrients in the grains is expected to contribute to better understand the magnitude of this interaction and the potential identification of stable genotypes for these traits. The present investigation was carried out during kharif 2013 and 2014 in the experimental farm of Zonal Agricultural Research Station (ZARS), Bengaluru and ZARS V. C Farm Mandya to identify stable genotypes southern dry zone. The fourteen genotypes were evaluated at two locations to assess the genetic variability, detect genotype xenvironment interaction and identification of genotypes which are stable across the two locations for grain nutrient content. Analysis of variance for individual environment for grain nutrient content revealed highly significant differences among the genotypes. Genotypes JWM-1 and VR-1034 for calcium content; OUAT-2, TNEC-1234 and GPU-71 for iron content and OUAT-2, TNEC-1234, VR-1034, GPU-71 and VR-936 for zinc content were identified. Pooled analysis of variance showed significant genotype $\times$ environment effects were observed for grain Calcium, iron and zinc contents. Eberhart and Russel stability parameters indicated the genotypes OUAT-2, VR-1034, GPU-71, GE-728 and JWM-1 were consistently adapted across the environments whereas, TNEC-1234, VR-936, GE-6834-1, VL-384, WFM-10, KMR-344, DHWFM 11-3, DHWFM 23 and GPU-67 were poorly adapted across the environments for their grain calcium content. Genotypes VR-1034, GPU-71, DHWFM 11-3, OUAT-2 and JWM-1were consistently stable across the environments whereas VR-936, GE-728, GE-6834-1, WFM-10, KMR-344, DHWFM 2-3 and GPU-67 were poorly adapted across the environments for their grain iron content. The genotypes TNEC-1234, VR-936, and GPU-71 were consistently stable whereas OUAT-2, GE-728, GE-6834-1, VL-384, JWM-1, KMR-344, DHWFM 2-3, GPU-67 poorly stable across the environment for their grain zinc content. It is observed that genotype GPU-71 is stable for iron, calcium and zinc contents across the two locations and two years. This genotype could be used as a parent for hybridization to improve micronutrient content.
Common name "finger millet" is derived from the finger like branching of the panicle. The cultivated Eleusine coracana is an allotetraploid with chromosome number $2 n=4 x=36$. There is considerable polymorphism for grain colour in finger 
millet. Traditionally brown coloured grains are predominant and preferred by the consumers. Of late, white grains are preferred by the food processing industries because of their high protein, low tannins and increased consumer acceptability (Sharathbabu et al., 2008).

Finger millet is a nutritious food grain crop with a fair amount of protein (7.3g $100 \mathrm{~g}^{-1}$ ) (Malleshi and Klopfenstein, 1998), dietary fibre (15-20\%), (Chethan and Malleshi, 2007) and rich source of calcium (344 mg $100 \mathrm{~g}^{-1}$ ) (Gopalan et al., 2002). Wider adoptability (Uphadhayaya et al., 2007) and higher nutritional quality, higher multiplication rate and longer shelf life under ambient conditions (Iyengar et al., 1945), makes finger millet an ideal crop for use as a staple food and famine reserve. The most cost effective approach for mitigating micronutrient and protein malnutrition is to introduce finger millet varieties selected and/or bred for increased iron, zinc and protein contents through plant breeding. Attempts to breed finger millet for enhanced grain micro nutrient and protein contents are still in its infancy. As finger millet is a staple food crop of resource poor users and consumed in large quantities on daily basis, developing nutrient rich finger millet cultivars will contribute to reduced micro nutrient malnutrition.

\section{Materials and Methods}

The material for present investigation was obtained from Project Coordinating Unit (Small millets), Bengaluru under All India Coordinated Small Millets Improvement Project. Material consisted of fourteen genotypes of finger millet including one white finger millet and one brown finger millet check. List of genotypes used for the investigation is shown in table 1 . Experimental material was evaluated over two locations viz., Bengaluru, Mandya and two seasons Kharif 2013 and 2014. The experiment was laid out in simple Randomised Complete Block Design with three replications. The plot size at each location was $3 \mathrm{~m}$ length comprising of 10 rows and planting was done at a spacing of $22.5 \mathrm{~cm}$ between rows and $10 \mathrm{~cm}$ between two hills in a row maintaining one healthy and vigorous seedling per hill. Fertilizers were applied at recommended dose of 50:40:25 NPK kg per ha. Nitrogen was given in two splits 50 per cent as basal and 50 per cent as top dressing. Inter cultivation practices were followed in both the locations.

\section{Determination of grain nutrient concentrations}

The seeds of fourteen genotypes were thoroughly cleaned to remove foreign particles, samples were prepared from bulk, powdered, sieved through 40 mesh size and were used for estimation of grain nutrient content. The Protein content of the dried powder was estimated as per cent total nitrogen by micro Kjeldhal procedure and converted to per cent protein. Fat, fibre, Ash, Carbohydrate and total energy are determined according to AOAC, 1980 method. Mineral concentrations were determined by atomic absorption spectrophotometry (Perkin-Elmer 560), in an acetylene-air flame at the following wavelengths: $285 \mathrm{~nm}(\mathrm{Ca}), 248 \mathrm{~nm}$ (Fe) and $214 \mathrm{~nm}(\mathrm{Zn})$.

\section{Statistical analysis}

The statistical analysis of the data on the individual character was carried out on the mean value of five randomly selected plants on each genotype from each of the three replications. The analysis of variance for different Characters was carried out for each location separately in order to partition the variability due to different sources following the method given by Pense and Sukhatme (1964). 
The stability model proposed by Eberhart and Russell (1966) was adopted to analyze the data over four environments.

The model involves the estimation of three parameters, mean $(\mathrm{X})$, regression coefficient $\left(b_{i}\right)$ and deviation from regression $\left(S^{2} d_{i}\right)$, which are defined by the following mathematical formula.

$\mathrm{Y}_{\mathrm{ij}}=\mu_{\mathrm{i}}+\beta_{\mathrm{i}} \mathrm{I}_{\mathrm{j}}+\delta_{\mathrm{ij}}$

Where,

$Y_{i j}$ : Mean of the $i^{\text {th }}$ genotype at the $j^{\text {th }}$ location

$(i=1,2,3,4,5 \ldots .19, j=1,2,3)$

$\mu_{\mathrm{I}}$ : The mean of $i^{\text {th }}$ genotype over all the location

$\beta_{\mathrm{I}}$ : The regression coefficient that measures the response of $i^{\text {th }}$ genotype to varying location

$\delta_{\mathrm{ij}}$ : The deviation from regression of the $\mathrm{i}^{\text {th }}$ genotype of $\mathrm{j}^{\text {th }}$ location

$I_{j}$ : The location index obtained as the deviation from the mean of all the genotypes at the $\mathrm{j}^{\text {th }}$ environment from the grand mean.

Mean $\left(\mu_{\mathrm{i}}\right)$, regression coefficient $\left(b_{\mathrm{i}}\right)$ and mean square deviation from linear regression line $\left(S^{2} d_{i}\right)$ are the three stability parameters proposed by Eberhart and Russell (1966) in their stability model. These parameters were computed by using the following formulae.

Additive Main Effects and Multiplicative interaction effects (AMMI) model of analysis of variance for stability was followed for working out the variance for stability. The mathematical model for AMMI is

$\mathrm{Y}_{\mathrm{ger}}=\mu+\alpha_{\mathrm{g}}+\beta_{\mathrm{e}}+\Sigma \lambda_{\mathrm{n}} \gamma_{\mathrm{gn}} \delta_{\mathrm{en}}+\rho_{\mathrm{ge}}+\varepsilon_{\mathrm{ger}}$ $\mathrm{g}=$ Genotypes

$\mathrm{e}=$ Environments

$\mathrm{r}=$ replications

$\mathrm{Y}=$ character of genotype $\mathrm{g}$ in environment $\mathrm{e}$

$\mu=$ Grand mean

$\alpha_{\mathrm{g}}=$ Mean of the $\mathrm{g}^{\text {th }}$ genotype minus the grand mean

$\beta_{\mathrm{e}}=$ Mean of the $\mathrm{e}^{\text {th }}$ genotype minus the grand mean

$\mathrm{n}=$ Number of ICPAs (Interaction Principal Component axis) retained in the model.

$\lambda_{\mathrm{n}}=$ Singular value or square root of the Eigen value of the PCA axis $n$

$\gamma_{\mathrm{gn}}=$ principal component score for PCA axis of the $\mathrm{g}^{\text {th }}$ genotype

$\delta_{\text {en }}=$ Principal component score for PCA axis

' $n$ ' of the $\mathrm{e}^{\text {th }}$ environment

$\varepsilon_{\text {ger }}=$ Residual

\section{Results and Discussion}

The mean protein content of the genotypes was comparable in all environments (Table 2). The protein content was highest $(11.547 \%)$ and least $(6.69 \%)$ in seeds of GE-6834-1 and GPU-67, respectively across the four environments (Fig. 1). The genotypes VR1034 (9.69\%), GE-728 (9.58\%), VL$384(10.07 \%)$ and KMR-344 (9.02\%) had higher protein content than the experimental mean $(8.95 \%)$. However the check GPU$67(6.69 \%)$ had lower grain protein content in all environments. A wide range of protein content (6.6\% to $11.6 \%)$ was observed among the genotypes. The estimates of PCV and 
GCV were moderate across the environments whereas, higher heritability was noticed across the environments. Narrower differences were observed between PCV and GCV for protein content across the environments (Table 3 ).

The seeds of JWM-1 (422.35mg/100g) had higher calcium content while those of DHWFM 2-3 had lowest calcium content (168.41 mg/100g) (Fig. 2). Seven genotypes had higher calcium content than brown grained check (239.26mg/100g) (Fig 2). A wide range $(164 \mathrm{mg} / 100 \mathrm{~g}$ to $424 \mathrm{mg} / 100 \mathrm{~g})$ of calcium content was observed among the genotypes. The estimates of PCV and GCV were high for all environments. Genotypes exhibited higher heritability across the environments except during kharif 2014 at Mandya (56.92\%). Narrower differences were observed between PCV and GCV for calcium content across the environments (Table 3).

The seeds of genotype OUAT-2 had highest $(10.13 \mathrm{mg} / 100 \mathrm{~g})$ iron content followed by GPU-71 (9.87mg/100g) and TNEC-1234 (9.29 $\mathrm{mg} / 100 \mathrm{~g}$ ) and lowest was observed in GE-728 (2.90 mg/100g) (Fig. 2). A wide range (2.9 $\mathrm{mg} / 100 \mathrm{~g}$ to $10.4 \mathrm{mg} / 100 \mathrm{~g}$ ) of iron content was noticed among the genotypes. The estimates of PCV and GCV were high whereas heritability was high across the environments. Difference between PCV and GCV were narrow for iron content across the environments (Table 3).

The seeds of genotype OUAT-2 (6.64 $\mathrm{mg} / 100 \mathrm{~g}$ ) had highest zinc content followed by TNEC-1234 (5.82 mg/100g) and VR-1034 (5.71 mg/100g). Except VR-1034 and GE6834-1, the other genotypes have not exhibited variation for location and season (Fig. 3). Zinc content of the genotypes ranged from $2.55 \mathrm{mg} / 100 \mathrm{~g}$ to $6.88 \mathrm{mg} / 100 \mathrm{~g}$. The estimates of genetic parameters PCV and GCV were high across the locations and seasons. Zinc content of genotypes registered high heritability across the environments. Difference between PCV and GCV were narrow for zinc content across the environments (Table 3 ).

None of the genotypes were superior for all the traits. Nevertheless, the genotype OUAT-2 had higher iron and zinc content.

Substantial variability for all grain nutrients observed in the fourteen finger millet genotypes. The genotypes differed significantly for protein, calcium, iron and zinc content. Grain nutrient content among the genotypes were comparable across the environments. Genotypes viz., GE-6834-1, VL-384, VR-1034 and GE-728are promising for protein; JWM-1 and VR-1034 were promising for high calcium content.

White finger millet check OUAT-2 has a highest iron and zinc content among the fourteen genotypes. These genotypes can be utilized in hybridization with agronomically superior accessions/breeding lines to combine grain nutrients with farmer/consumer preferred traits. Similar findings have been reported in finger millet germplasm lines by Uphadhayaya et al., (2011a).

The estimates of PCV and GCV were high for calcium, iron and zinc content in all the environments indicating the low effects of environments and greater role of genetic factors on the expression of a the character which offer scope for direct selection. Similar findings were reported in calcium (Vadivoo et al., 1998), iron (Samak et al., 2011 in rice; Govindraj, 2011 in pearl millet) and zinc (Samak et al., 2011 in rice; Berhanu Dagnaw et al., 2013 in rice).Moderate PCV and GCV were recorded for the grain protein content and indicated fairly large extent of variation for this trait. This is in accordance with the findings of the Vadivoo et al., (1998) and Govindraj (2011). 
Fig.1 Mean performance of finger millet genotypes for grain calcium content across the four environments

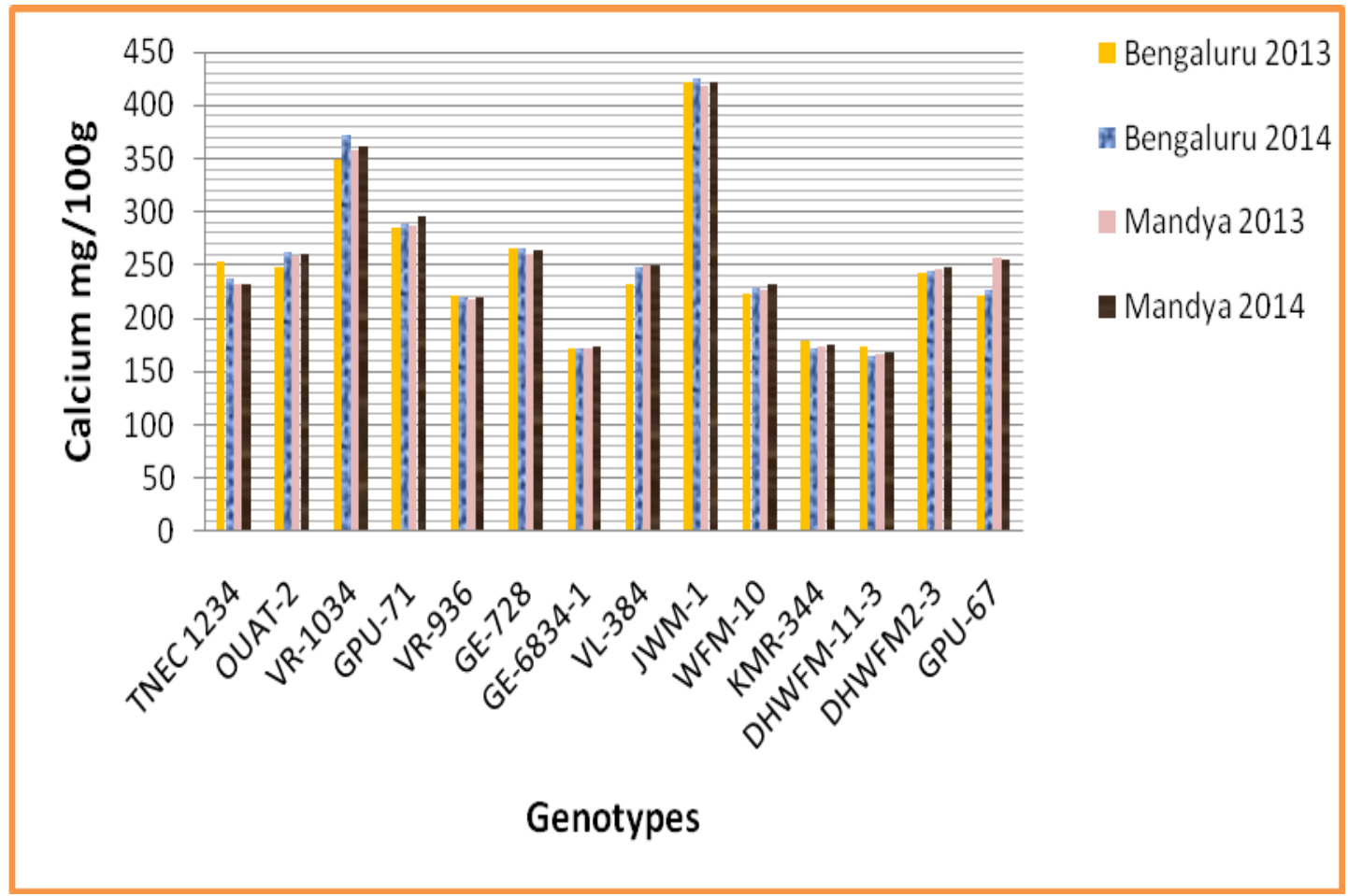

Fig.2 Mean performance of finger millet genotypes for grain iron content across the four environments

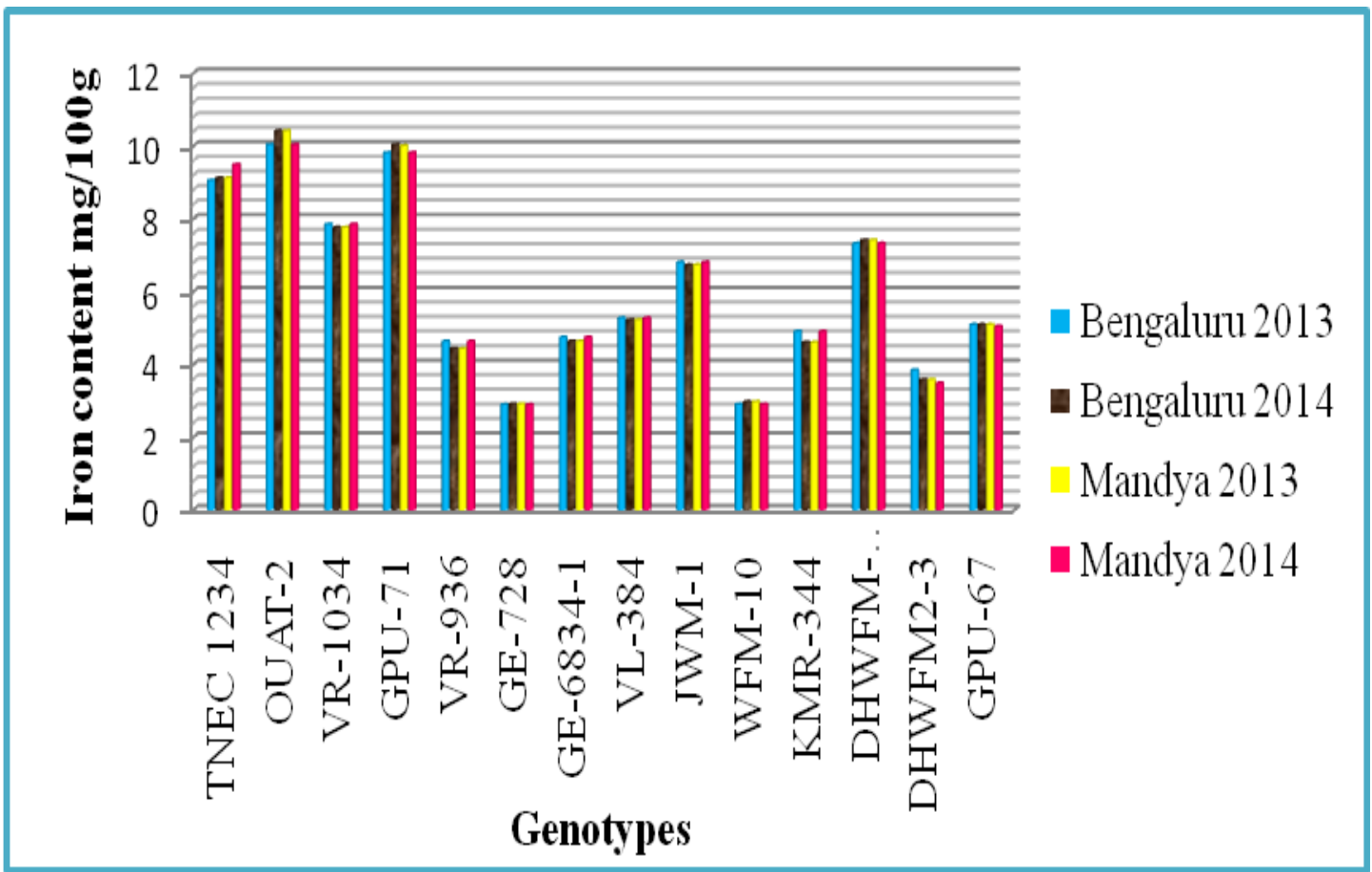


Fig.3 Mean performance of finger millet genotypes for grain zinc content across the four environments

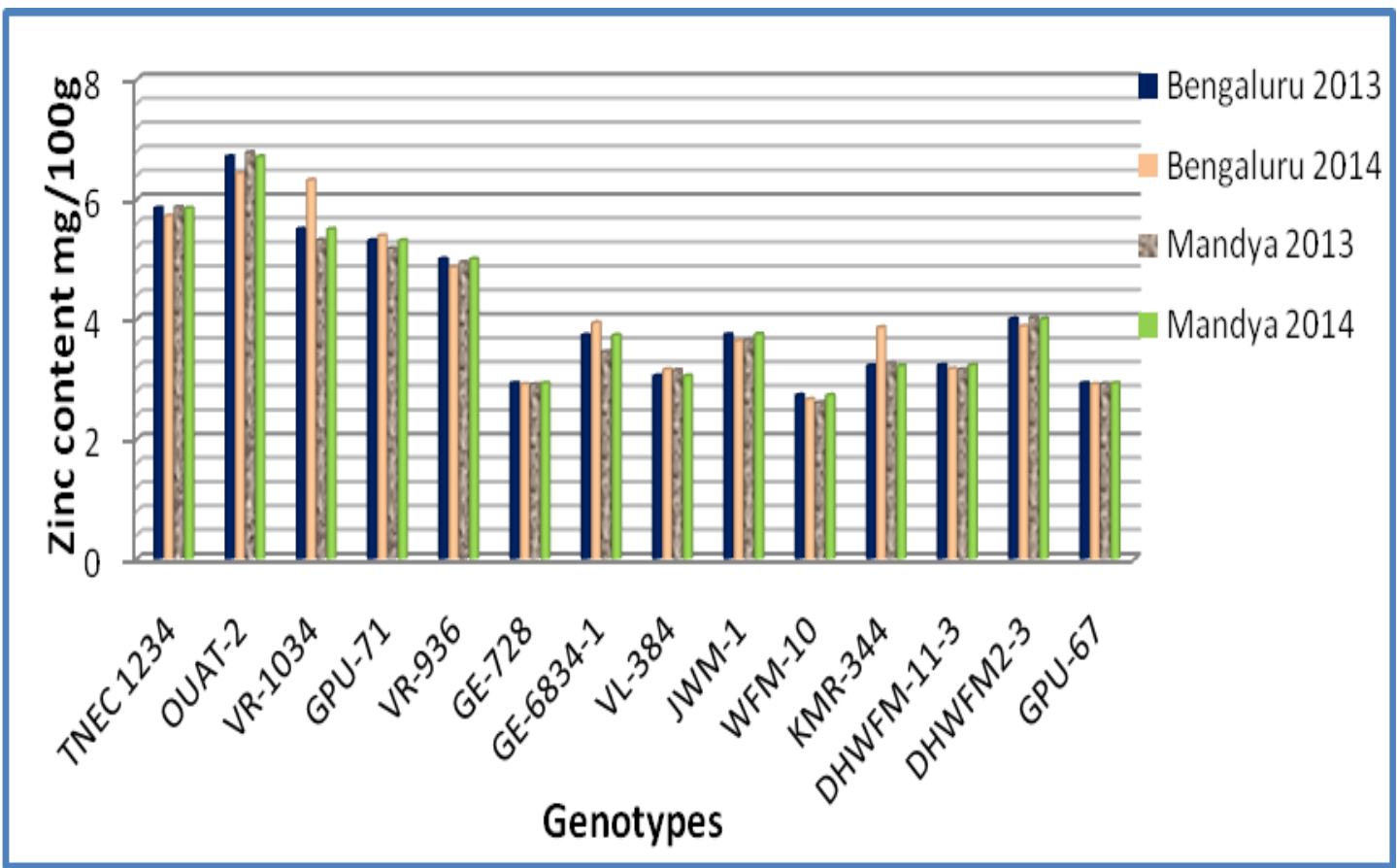

Fig.4 AMMI biplot of main effects and G x E interaction of white finger millet genotypes for grain calcium content over locations and year

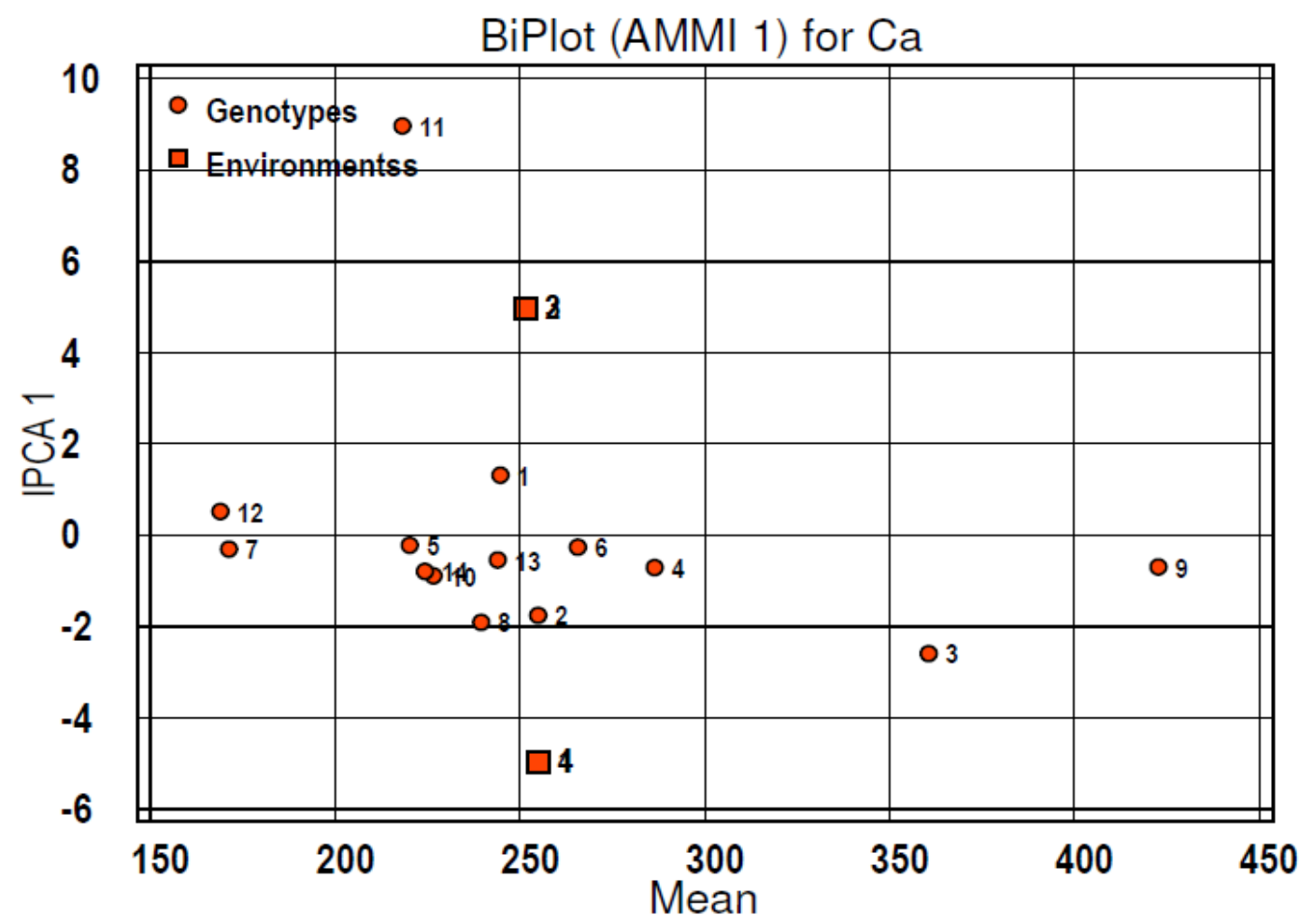


Fig.5 AMMI biplot of main effects and $\mathrm{G} x \mathrm{E}$ interaction of white finger millet genotypes for grain iron content over locations and years
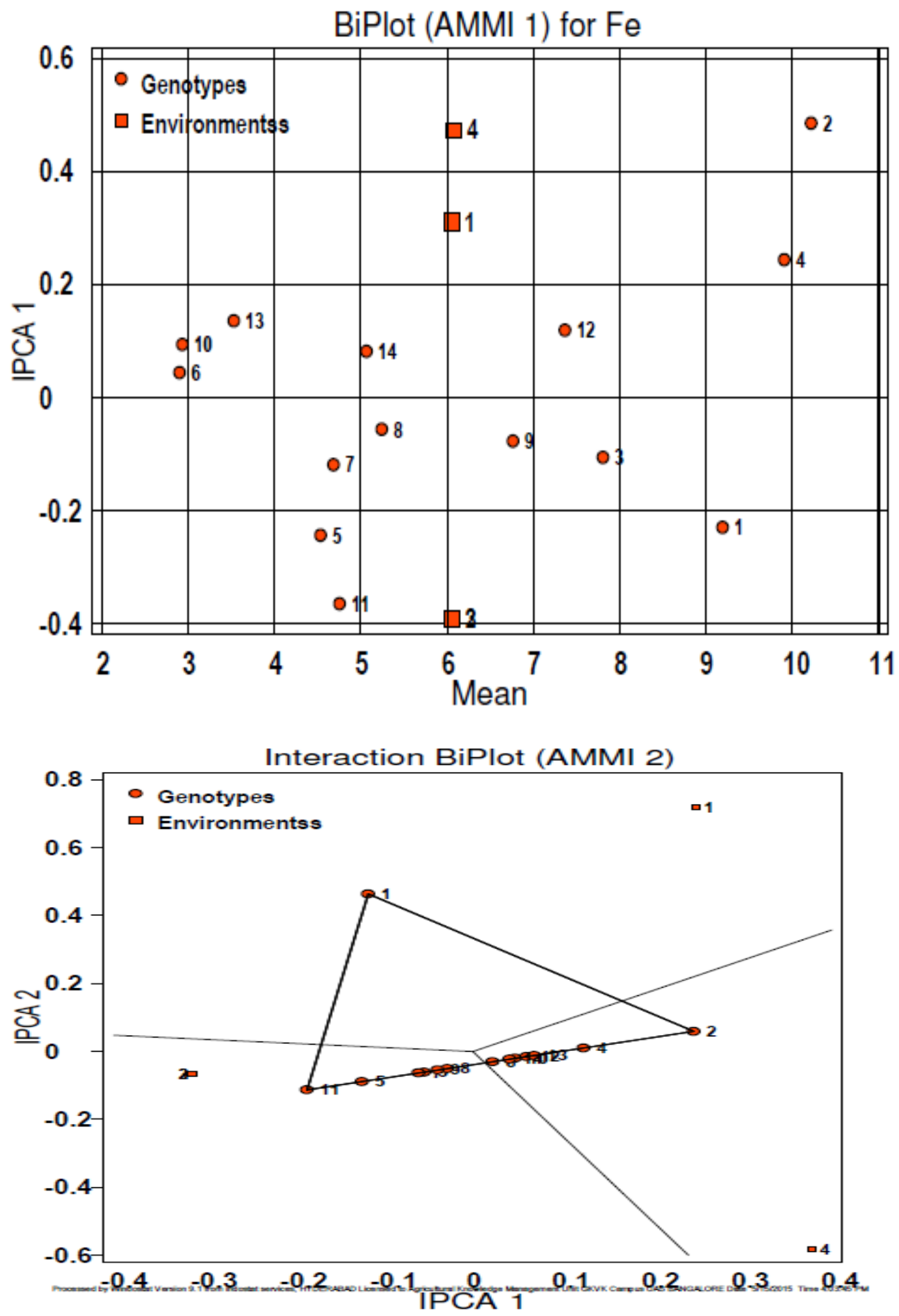
Fig.6 AMMI biplot of main effects and $\mathrm{G} x \mathrm{E}$ interaction of white finger millet genotypes for grain zinc content over locations and years
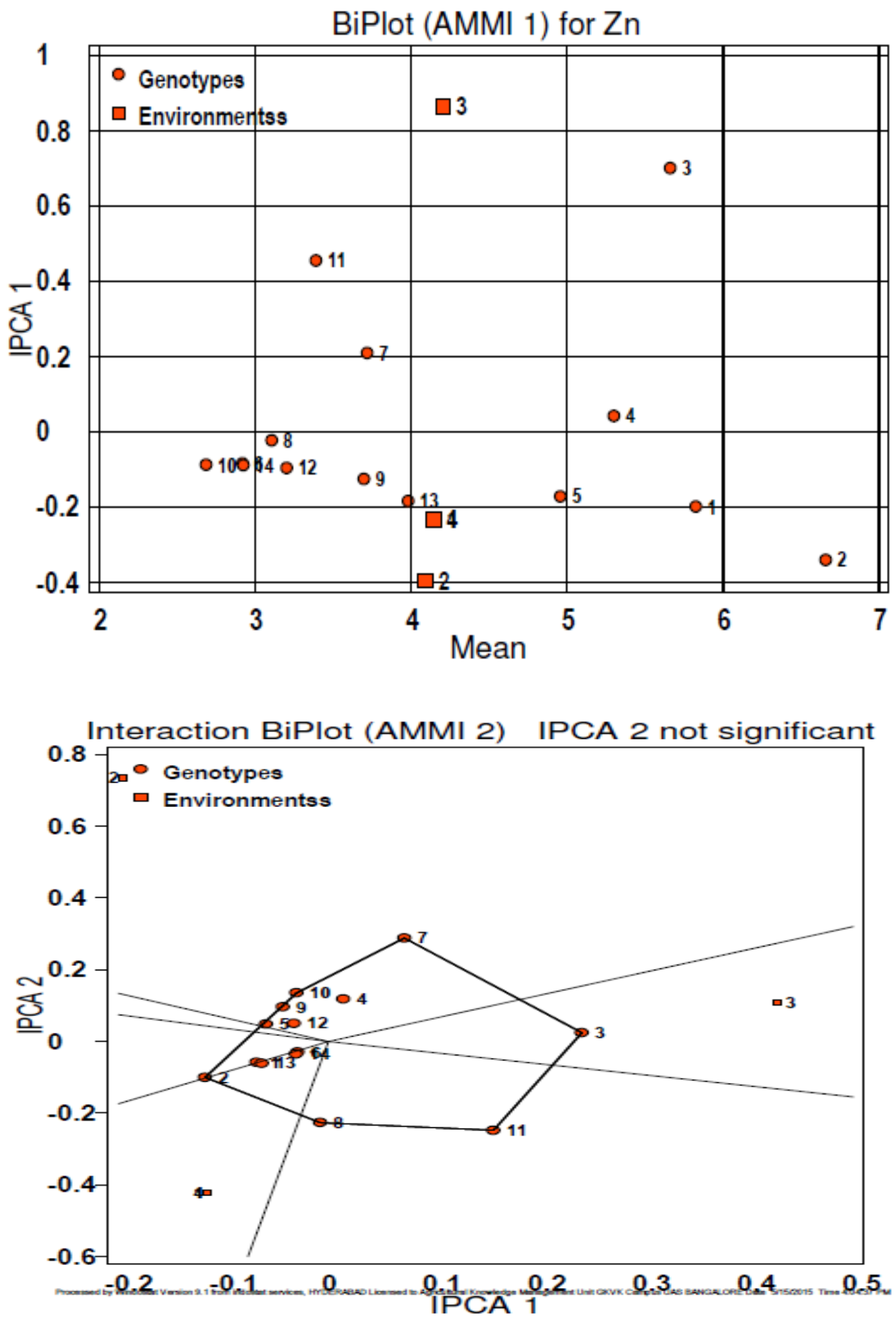
Table.1 List of genotypes and their pedigree

\begin{tabular}{|c|c|c|}
\hline Varieties & Pedigree & Origin \\
\hline TNEC-1234 & Co-9 x GE-47 & Coimbatore, Tamil nadu \\
\hline OUAT-2 (Suvra) & Selection from Co-9 & Berhampur, Odisha \\
\hline VR-1034 & Unknown & Vizianagaram, A.P \\
\hline GPU-71 & GE-4971 x VL 147 & Bangalore, Karnataka \\
\hline VR-936 (Hima) & IE 2695 x Godavari (PR-202) & Vizianagaram, A.P \\
\hline GE-728 & Germplasm line & Unknown \\
\hline GE-6834-1 & Germplasm line & Unknown \\
\hline VL-384 & OUAT $2 \times$ GEC- 450 & Almora, Uttarkhand \\
\hline JWM-1 & Unknown & Jagdalpur, Chattisgarh \\
\hline WFM-10 & $\begin{array}{l}\text { Pureline selection derived from } \\
\text { GPU- } 28 \text { x GE- } 4985\end{array}$ & Bangalore, Karnataka \\
\hline KMR-344 & WRC-1 x Indaf-11 & Mandya, Karnataka \\
\hline DHWFM 11-3 & $\begin{array}{l}\text { Pureline selection from WFM- } \\
\qquad 11\end{array}$ & Hanumanamatti, Karnataka \\
\hline DHWFM 2-3 & Pureline selection from WFM-2 & Hanumanamatti, Karnataka \\
\hline GPU-67* & Selection from GE-5331 & Bangalore, Karnataka \\
\hline
\end{tabular}


Table.2 Mean performances of white finger millet genotypes for grain nutrient content across two locations and two years

\begin{tabular}{|c|c|c|c|c|c|c|c|c|c|c|c|c|c|c|}
\hline \multirow[t]{3}{*}{ Genotype } & \multicolumn{7}{|c|}{ Protein (\%) } & \multicolumn{7}{|c|}{ Calcium (mg/100g) } \\
\hline & \multicolumn{2}{|c|}{ Bengaluru } & \multirow[b]{2}{*}{ Mean } & \multicolumn{2}{|c|}{ Mandya } & \multirow[b]{2}{*}{ Mean } & \multirow{2}{*}{$\begin{array}{l}\text { Grand } \\
\text { mean }\end{array}$} & \multicolumn{2}{|c|}{ Bengaluru } & \multirow[b]{2}{*}{ Mean } & \multicolumn{2}{|c|}{ Mandya } & \multirow[b]{2}{*}{ Mean } & \multirow{2}{*}{$\begin{array}{c}\text { Grand } \\
\text { mean }\end{array}$} \\
\hline & 2013 & 2014 & & 2013 & 2014 & & & 2013 & 2014 & & 2013 & 2014 & & \\
\hline $\begin{array}{l}\text { TNEC- } \\
1234 \\
\end{array}$ & 7.86 & 7.847 & 7.86 & 7.847 & 7.87 & 7.86 & 7.857 & 253.02 & 236.60 & 244.81 & 231.21 & 232.03 & 231.62 & 238.42 \\
\hline OUAT-2 & 8.97 & 8.967 & 8.97 & 8.967 & 8.96 & 8.97 & 8.968 & 247.82 & 262.00 & 254.91 & 258.03 & 260.65 & 259.34 & 257.78 \\
\hline VR-1034 & 9.72 & 9.66 & 9.69 & 9.66 & 9.77 & 9.69 & 9.693 & 349.42 & 371.91 & 360.67 & 357.53 & 362.12 & 359.83 & 361.39 \\
\hline GPU-71 & 8.82 & 8.853 & 8.84 & 8.853 & 8.82 & 8.84 & 8.837 & 284.54 & 288.31 & 286.43 & 287.69 & 296.11 & 291.90 & 291.27 \\
\hline VR-936 & 7.817 & 7.877 & 7.85 & 7.877 & 7.87 & 7.85 & 7.847 & 220.87 & 219.68 & 220.28 & 217.21 & 219.41 & 218.31 & 219.84 \\
\hline GE-728 & 9.587 & 9.557 & 9.57 & 9.577 & 9.57 & 9.58 & 9.582 & 266.11 & 265.45 & 265.78 & 260.49 & 264.87 & 262.68 & 265.33 \\
\hline $\begin{array}{l}\text { GE-6834- } \\
1\end{array}$ & 11.54 & $\begin{array}{c}11.64 \\
7\end{array}$ & 11.60 & $\begin{array}{c}11.44 \\
7\end{array}$ & 11.54 & 11.50 & 11.547 & 171.42 & 171.19 & 171.31 & 172.10 & 172.80 & 172.45 & 172.05 \\
\hline VL-384 & 10.04 & $\begin{array}{c}10.09 \\
7\end{array}$ & 10.07 & $\begin{array}{c}10.09 \\
7\end{array}$ & 10.47 & 10.07 & 10.072 & 231.78 & 247.47 & 239.63 & 249.69 & 249.77 & 249.73 & 244.70 \\
\hline JWM-1 & 8.57 & 8.56 & 8.57 & 8.56 & 8.47 & 8.57 & 8.565 & 420.91 & 424.55 & 422.73 & 417.81 & 421.96 & 419.89 & 422.35 \\
\hline WFM-10 & 8.94 & 8.86 & 8.90 & 8.86 & 8.84 & 8.90 & 8.9 & 223.81 & 229.37 & 226.59 & 226.67 & 231.68 & 229.18 & 229.14 \\
\hline KMR-344 & 9.023 & 9 & 9.01 & 9 & 9.23 & 9.01 & 9.02 & 264.47 & 171.95 & 218.21 & 173.69 & 174.51 & 174.10 & 196.36 \\
\hline $\begin{array}{l}\text { DHWFM } \\
\mathbf{1 1 - 3}\end{array}$ & 8.933 & 8.947 & 8.94 & 8.947 & 8.9 & 8.94 & 8.94 & 173.27 & 164.74 & 169.01 & 166.22 & 167.82 & 167.02 & 168.41 \\
\hline $\begin{array}{l}\text { DHWFM } \\
2-3\end{array}$ & 8.833 & 8.9 & 8.87 & 8.9 & 8.83 & 8.87 & 8.867 & 242.96 & 245.04 & 244.00 & 245.97 & 248.63 & 247.30 & 246.32 \\
\hline GPU-67 & 6.63 & 6.763 & 6.70 & 6.763 & 6.63 & 6.70 & 6.697 & 221.94 & 226.53 & 224.24 & 256.80 & 254.28 & 255.54 & 239.26 \\
\hline
\end{tabular}




\begin{tabular}{|c|c|c|c|c|c|c|c|c|c|c|c|c|c|c|}
\hline \multirow[t]{3}{*}{ Genotype } & \multicolumn{7}{|c|}{ Iron (mg/100g) } & \multicolumn{7}{|c|}{ Zinc (mg/100g) } \\
\hline & \multicolumn{2}{|c|}{ Bengaluru } & \multirow{2}{*}{ Mean } & \multicolumn{2}{|c|}{ Mandya } & \multirow{2}{*}{ Mean } & \multirow[t]{2}{*}{ Grand mean } & \multicolumn{2}{|c|}{ Bengaluru } & \multirow[b]{2}{*}{ Mean } & \multicolumn{2}{|c|}{ Mandya } & \multirow{2}{*}{ Mean } & \multirow[t]{2}{*}{ Grand mean } \\
\hline & 2013 & 2014 & & 2013 & 2014 & & & 2013 & 2014 & & 2013 & 2014 & & \\
\hline TNEC-1234 & 9.05 & 9.12 & 9.09 & 9.12 & 9.49 & 9.31 & 9.29 & 5.85 & 5.72 & 5.79 & 5.87 & 5.85 & 5.86 & 5.82 \\
\hline OUAT-2 & 10.03 & 10.41 & 10.22 & 10.41 & 10.03 & 10.22 & 10.13 & 6.71 & 6.43 & 6.57 & 6.78 & 6.71 & 6.75 & 6.64 \\
\hline VR-1034 & 7.85 & 7.76 & 7.81 & 7.76 & 7.85 & 7.81 & 7.83 & 5.5 & 6.32 & 5.91 & 5.32 & 5.5 & 5.41 & 5.71 \\
\hline GPU-71 & 9.81 & 10.03 & 9.92 & 10 & 9.81 & 9.91 & 9.87 & 5.31 & 5.39 & 5.35 & 5.18 & 5.31 & 5.25 & 5.33 \\
\hline VR-936 & 4.63 & 4.43 & 4.53 & 4.43 & 4.63 & 4.53 & 4.58 & 5 & 4.86 & 4.93 & 4.95 & 5 & 4.98 & 4.97 \\
\hline GE-728 & 2.89 & 2.91 & 2.90 & 2.91 & 2.89 & 2.90 & 2.90 & 2.93 & 2.91 & 2.92 & 2.91 & 2.93 & 2.92 & 2.93 \\
\hline GE-6834-1 & 4.74 & 4.63 & 4.69 & 4.63 & 4.74 & 4.69 & 4.71 & 3.73 & 3.94 & 3.84 & 3.46 & 3.73 & 3.60 & 3.78 \\
\hline VL-384 & 5.27 & 5.22 & 5.25 & 5.22 & 5.27 & 5.25 & 5.26 & 3.05 & 3.16 & 3.11 & 3.15 & 3.05 & 3.10 & 3.08 \\
\hline JWM-1 & 6.8 & 6.73 & 6.77 & 6.73 & 6.8 & 6.77 & 6.78 & 3.74 & 3.64 & 3.69 & 3.65 & 3.75 & 3.70 & 3.72 \\
\hline WFM-10 & 2.9 & 2.97 & 2.94 & 2.97 & 2.9 & 2.94 & 2.92 & 2.73 & 2.66 & 2.70 & 2.61 & 2.73 & 2.67 & 2.71 \\
\hline KMR-344 & 4.9 & 4.6 & 4.75 & 4.6 & 4.9 & 4.75 & 4.83 & 3.22 & 3.86 & 3.54 & 3.26 & 3.22 & 3.24 & 3.38 \\
\hline DHWFM 11-3 & 7.32 & 7.41 & 7.37 & 7.41 & 7.32 & 7.37 & 7.34 & 3.23 & 3.17 & 3.20 & 3.16 & 3.23 & 3.20 & 3.22 \\
\hline DHWFM 2-3 & 3.84 & 3.58 & 3.71 & 3.58 & 3.48 & 3.53 & 3.60 & 4.02 & 3.88 & 3.94 & 4.02 & 4.03 & 4.01 & 3.97 \\
\hline GPU-67 & 5.09 & 5.09 & 5.09 & 5.09 & 5.04 & 5.07 & 5.07 & 2.93 & 2.91 & 2.92 & 2.92 & 2.93 & 2.93 & 2.93 \\
\hline
\end{tabular}

Table.3 Estimates of genetic components in white finger millet genotypes for grain nutrient contents in different locations

\begin{tabular}{|c|c|c|c|c|c|c|c|c|c|c|c|c|c|c|c|c|}
\hline \multirow[t]{3}{*}{ Traits } & \multicolumn{4}{|c|}{ Range } & \multicolumn{4}{|c|}{ PCV (\%) } & \multicolumn{4}{|c|}{ GCV (\%) } & \multicolumn{4}{|c|}{$\mathrm{H}^{2}(\mathrm{bs})(\%)$} \\
\hline & \multicolumn{2}{|c|}{ Bengaluru } & \multicolumn{2}{|c|}{ Mandya } & \multicolumn{2}{|c|}{ Bengaluru } & \multicolumn{2}{|c|}{ Mandya } & \multicolumn{2}{|c|}{ Bengaluru } & \multicolumn{2}{|c|}{ Mandya } & \multicolumn{2}{|c|}{ Bengaluru } & \multicolumn{2}{|c|}{ Mandya } \\
\hline & 2013 & 2014 & 2013 & 2014 & 2013 & 2014 & 2013 & 2014 & 2013 & 2014 & 2013 & 2014 & 2013 & 2014 & 2013 & 2014 \\
\hline $\mathbf{P}$ & $6.77-11.07$ & $6.83-11.6$ & $6.6-11.5$ & $6.6-11.6$ & 13.1 & 12.6 & 12.53 & 12.7 & 12.87 & 12.4 & 12.45 & 12.76 & 96.5 & 96.7 & 98.75 & 26.27 \\
\hline $\mathrm{Ca}$ & $171.2-424$ & $171.4-420$ & $164-424$ & $169-422$ & 28.9 & 25.8 & 27.54 & 27.7 & 28.89 & 25.4 & 27.49 & 27.68 & 99.6 & 96.50 & 99.6 & 56.92 \\
\hline $\mathrm{Fe}$ & 2.91-10.04 & $2.90-10.0$ & 2.91-10.4 & $2.9-10$ & 41.6 & 41.0 & 40.92 & 41.1 & 41.65 & 40.8 & 40.82 & 40.9 & 99.8 & 98.8 & 99.5 & 84.00 \\
\hline $\mathbf{Z n}$ & $2.55-6.8$ & $2.55-6.88$ & $2.61-6.78$ & $2.9-6.66$ & 32.4 & 32.4 & 31.74 & 30.9 & 32.40 & 32.4 & 31.645 & 30.27 & 99.85 & 99.8 & 99.3 & 61.04 \\
\hline
\end{tabular}


Table.4 Analysis of variance for white finger millet genotypes for grain nutrient characters over locations and years

P- Protein Ca- Calcium Fe- Iron Zn- Zinc content

\begin{tabular}{|l|}
\multicolumn{1}{|c|}{ Source of variation } \\
\hline Location \\
\hline Year \\
\hline Location $\times$ year \\
\hline Genotype \\
\hline Location $\times$ genotype \\
\hline Genotype $\times$ year \\
\hline Genotype $\times$ year $\times$ location \\
\hline Error \\
\hline
\end{tabular}

\begin{tabular}{|c|c|c|c|c|}
\hline \multirow{2}{*}{ Df } & & \multicolumn{4}{|c|}{ Mean sum of squares } \\
\hline 1 & $\mathbf{P}$ & Ca & Fe & Zn \\
\hline 1 & 0.078 & 20.136 & 0.113 & 0.0002 \\
\hline 1 & 0.0767 & $369.54 * *$ & 0.0005 & 0.1074 \\
\hline 13 & $15.225 * *$ & $55934.52 * *$ & $73.912 * *$ & $20.028 * *$ \\
\hline 13 & 0.0153 & $759.21 * *$ & $0.1254 * *$ & $0.079 * *$ \\
\hline 13 & 0.0164 & $544.03 * *$ & $0.0102 * *$ & 0.0427 \\
\hline 13 & 0.0466 & $595.09 * *$ & 0.0732 & 0.0513 \\
\hline 112 & 0.0359 & 47.325 & 0.0396 & 0.0277 \\
\hline
\end{tabular}

$*$ Significance at 0.05 probability ** Significance at 0.01 probability

Table.5 AMMI ANOVA of white finger millet genotypes for grain nutrient content traits

\begin{tabular}{|c|c|c|c|c|}
\hline \multicolumn{2}{|c|}{ Source of variation } & \multicolumn{3}{|c|}{ Mean Sum of Squares } \\
\hline Traits & df & $\mathrm{Ca}$ & Fe & Zn \\
\hline Genotype & 13 & $18321.15^{* *}$ & $24.893 * *$ & $6.62 * *$ \\
\hline Environments & 3 & 53.777 & 0.0024 & 0.0309 \\
\hline G*E Interaction & 39 & 250.754 & 0.0121 & 0.028 \\
\hline PCA 1 & 15 & $651.96 * *$ & $0.0260 *$ & 0.068 \\
\hline PCA 2 & 13 & 0.00 & 0.0064 & 0.0058 \\
\hline Pooled residual & 24 & 250.75 & 0.0121 & 0.003 \\
\hline \multicolumn{2}{|c|}{$\%$ contribution of PCA 1} & $100 \%$ & $82.44 \%$ & $93.08 \%$ \\
\hline \multicolumn{2}{|c|}{$\%$ contribution of PCA 2} & $0.00 \%$ & $17.58 \%$ & $6.92 \%$ \\
\hline \multicolumn{2}{|c|}{ Residual } & $0.00 \%$ & $0.02 \%$ & $0.00 \%$ \\
\hline
\end{tabular}

Ca- Calcium Fe- Iron Zn- Zinc content

$*$ Significance at 0.05 probability ** Significance at 0.01 probability 
Table.6 Eberhart and Russel stability parameters for yield and its attributing traits in white finger millet genotypes

\begin{tabular}{|c|c|c|c|c|c|c|c|c|c|}
\hline \multirow[t]{2}{*}{ Genotype } & \multicolumn{3}{|c|}{ Calcium (mg/100g) } & \multicolumn{3}{|c|}{ Iron (mg/100g) } & \multicolumn{3}{|c|}{ Zinc (mg/100g) } \\
\hline & Mean & $\mathbf{b}_{\mathbf{i}}$ & $S^{2} d_{i}$ & Mean & $\mathbf{b}_{\mathbf{i}}$ & $\mathbf{S}^{2} \mathbf{d}_{\mathbf{i}}$ & Mean & $\mathbf{b}_{\mathbf{i}}$ & $\mathbf{S}^{2} \mathbf{d}_{\mathrm{i}}$ \\
\hline TNEC-1234 & 244.81 & 4.83 & -25.69 & 9.19 & 4.750 & -0.006 & 5.52 & -1.40 & -0.009 \\
\hline OUAT-2 & 254.91 & -4.17 & -25.67 & 10.22 & -3.523 & $0.059 *$ & 6.60 & -3.12 & -0.007 \\
\hline VR-1034 & 360.67 & -6.62 & -25.70 & 7.81 & 0.845 & -0.005 & 5.66 & 8.99 & $-0.024^{*}$ \\
\hline GPU-71 & 286.42 & -1.11 & -25.60 & 9.91 & -1.740 & 0.006 & 5.30 & 1.77 & -0.010 \\
\hline VR-936 & 220.27 & 0.35 & -25.65 & 4.58 & 1.865 & 0.009 & 4.95 & -0.83 & -0.006 \\
\hline GE-728 & 265.78 & 0.19 & -25.65 & 2.90 & -0.252 & 0.009 & 2.92 & -0.03 & -0.010 \\
\hline GE-6834-1 & 171.30 & 0.06 & -25.68 & 4.68 & 0.943 & -0.009 & 3.72 & 4.12 & -0.009 \\
\hline VL-384 & 239.63 & -4.62 & -25.66 & 5.24 & 0.481 & -0.004 & 3.10 & 0.15 & -0.005 \\
\hline JWM-1 & 422.73 & -1.07 & -25.70 & 6.76 & 0.632 & -0.008 & 3.69 & -0.18 & -0.066 \\
\hline WFM-10 & 226.59 & -1.63 & -27.60 & 2.93 & -0.620 & -0.007 & 2.68 & 0.35 & -0.005 \\
\hline KMR-344 & 218.21 & 27.52 & -25.66 & 4.15 & 2.756 & -0.033 & 3.39 & 5.51 & -0.034 \\
\hline DHWFM 11-3 & 169.00 & 2.51 & -25.52 & 7.36 & -0.813 & -0.006 & 3.20 & 0.04 & -0.009 \\
\hline DHWFM 2-3 & 244.00 & -0.61 & -25.65 & 3.53 & -0.929 & -0.003 & 3.98 & -1.25 & -0.010 \\
\hline GPU-67 & 224.24 & -1.35 & -25.60 & 5.06 & -0.532 & -0.008 & 2.92 & -0.10 & -6.010 \\
\hline
\end{tabular}

Table.7 Estimates of environmental indices for grain nutrient traits in finger millet

\begin{tabular}{|c|c|c|c|c|}
\hline Trait & Environment 1 & Environment 2 & Environment 3 & Environment 4 \\
\hline Calcium & 1.69 & 1.53 & 1.54 & 1.71 \\
\hline Iron & -0.013 & -0.002 & -0.0003 & 0.018 \\
\hline Zinc & -0.003 & -0.054 & 0.061 & -0.003 \\
\hline
\end{tabular}

Environment 1-----kharif 2013 at Bengaluru

Environment 2-----kharif 2013 at Mandya

Environment 3-----kharif 2014 at Bengaluru

Environment 4------kharif 2014 at Mandya 
Analysis of variance revealed highly significant differences among the genotypes for protein, calcium, iron and zinc content across the four environments. Analysis of variance indicated significant mean squares due to genotypes; genotype $\times$ environment interaction, genotype $\times$ year, location $\times$ year and genotype $\times$ location $\times$ year were significant for calcium. The mean sum of squares attributable to genotype, location $\times$ genotype and genotype $x$ year were significant for grain iron content. Analysis of variance revealed significant variation among genotypes and location $\times$ genotype interaction for grain zinc content. Maximum variation towards total variation is contributed by genotypes alone for grain protein, calcium, iron and zinc content (Table 4).

Environmental indices of kharif 2013 at Bengaluru and Mandya were positive for grain calcium content. During 2014 at Bengaluru environmental indices were positive for calcium and zinc content while they were negative during kharif 2014 at Mandya for grain zinc content (Table 7).

AMMI analysis of variance indicated significant mean squares due to PCA1 for calcium, iron and zinc content (Table 5). First principal component axis explains $100 \%$, $82.44 \%$ and $93.08 \%$ genotype $\times$ environment interaction for calcium, iron and zinc content, respectively.

The regression coefficient of genotypes OUAT-2, VR-1034, GPU-71, GE-728 and JWM-1 was highly non-significant coupled with high grain calcium content and nonsignificant deviation from regression indicating they were consistently adapted across the environments. On the other hand TNEC-1234, VR-936, GE-6834-1, VL-384, WFM-10, KMR-344, DHWFM 11-3, DHWFM 2-3 and GPU-67 exhibited lower grain calcium content compared to overall mean $(253.504 .41 \mathrm{mg} / 100 \mathrm{~g})$ with their unit regression coefficient and non-significant deviation from regression (Table 6) inferring they were poorly adapted across the environments which was in conformity with AMMI biplot analysis (Fig. 4).

For mean grain iron content, the genotypes VR-1034, GPU-71, DHWFM 11-3 and JWM1 had higher values than that of overall mean $(6.06 \mathrm{mg} / 100 \mathrm{~g})$ indicated they were consistently stable across the environments. On the other hand, genotypes VR-936, GE728, GE-6834-1, WFM-10, KMR-344, DHWFM 2-3 and GPU-67 had lower calcium content compared to overall mean coupled with non-significant regression coefficient and deviation from regression showing they were poorly adapted across the environments for their grain iron content (Table 6). Interaction biplot analysis of AMM sowed that the genotypes KMR-344, VR-936, WFM10, JWM-1 and VL-384 are best suitable genotypes during kharif 2013 at Mandya, genotype TNEC-1234 is best suited during kharif 2013 at Bengaluru and genotypes OUAT-2, GPU-71, GPU-67 and DHWFM 23 are best suited during kharif 2014 at Mandya (Fig. 5).

The seeds of genotypes TNEC-1234, VR-936, and GPU-71 recoded higher grain zinc content inferred they were consistently stable. The genotypes OUAT-2, GE-728, GE-68341, VL-384, JWM-1, KMR-344, DHWFM 2-3, GPU-67 and DHWFM 11-3 recorded lower grain zinc content compared to the overall mean $(4.41 \mathrm{mg} / 100 \mathrm{~g})$ indicating their poor stability across the environment for their grain zinc content. Besides this, all these genotypes manifested highly non-significant regression coefficient and deviation from the regression. The genotype VR-1034 had higher grain zinc content with significant $S^{2} d_{i}$ (Table 6). AMMI biplot interaction showed that the genotypes VR-1034 is best genotype during kharif 2014 
at Bengaluru, genotypes WFM-10, GE-68341, GPU-71 and JWM-1 are best suited genotypes during kharif 2013 at Mandya and genotypes TNEC-1234, DHWFM 2-3 and OUAT-2 are best suited at kharif 2014 at Mandya (Fig. 6).

To summarize it is observed that genotype GPU-71 is stable for iron, calcium and zinc contents across the two locations and two years. This genotype could be used as a parent for hybridization to improve micronutrient content.

\section{References}

AOAC, 1980, Official methods of analysis, 13th edition, Association of Official Analytical Chemists, Washington, DC.

BerhanuDagnaw, B., Rakhia, S., Naveen, G. K., Kundur, P. J. and Shashidhar, H. E., 2013, Estimation of genetic variability and correlation studies for grain zinc concentrations and yield related traits in selected rice (Oryza sativa L.) genotypes. Asian J. Exp. Biol. Sci., 4 (3): 391-397.

Chethan, S. and Malleshi, N.G., 2007. Finger millet poly phenols: characterization and their nutraceutical potential. Am. J. Food Technol., 2: 618-629.

Eberhart, S. A. and Russel, W. L., 1966, Stability parameters for components varieties.Crop Sci., 6: 36-40

Ganapathy, S., Nirmalakumari, A. and Muthiah, A.R., 2011, Genetic variability and inter relationship analyses form economic traits in finger millet germplasm. World J Agric Sci., 7 (2): 185-188.

Gopalan, C., Rama Sastri, B.V. and Balasubramanian, S.C., 2002. Nutritive Value of Indian Foods. National Institute of Nutrition, Indian Council of Medical Research, Hyderabad, India
Govindaraj, M., Selvi, B., Rajarathinam, S. and Sumathi, P., 2011a, Genetic variability and heritability of grain yield components and grain mineral concentration in India's pearl millet (Pennisetum glaucum) accessions. African J. Food. Agric. Nutri. Develop., 11 (3): 4758-4771.

Govindraj, J. M., 2011, Genetics of grain iron and zinc concentration in pearl millet (Pannisetum glaucum (L.) R. Br.) germplasm. Ph. D. Thesis, Tamil Nadu. Agril. Univ., Coimbatore.

Iyengar, K.G., Doraisami, L.S. and Iyengar, R.S., 1945. Ragi (Eleusine coracana). Mysore J. Agric. Sci., 24:33.

Malleshi., N. G. and Klopfenstein, C. F., 1998, Nutrient composition, amino acid and vitamin $\mathrm{c}$ intents of malted sorghum, pearl millet, finger millet and their rootlets. Int. J. Food Sci. Nutr., 49: 415-422.

Pense, V. G. and Sukhatme, D. V., 1964, Statistical methods for Agricultural Workers. Indian Council of Agricultural Research, Publication, New Delhi, p.115.

Samak, A., N. R., Hittalmani, S., Shashidhar, N. And Hanumareddy Biradar, 2011, Exploratory studies on genetic variability and genetic control for protein and micronutrient content in $\mathrm{F}_{4}$ and $\mathrm{F}_{5}$ generation of rice (Oryza sativa L.). Asian. J. Plt. Sci., 10 (7): 376-379.

Sharathbabu, K. S., Shanthakumar, G. and Salimath, P. M., 2008, Genetic variability and character association studies in white Ragi (Elusine coracana L. Gaertn). Kar.J.Agric.Sci., 21(4): 572575.

Upadhyaya, H. D., Ramesh, S., Shivali Sharma, Singh, S. K., Varshney, S. K., Sarma, N. D. R. K., Ravishankar, C. R., Narasimhudu, Y., Reddy, V. G., Sahrawata, K. L., Dhanalakshmi, T. N., Mgonja, M. A., Parziesh, H. K., Gowda, 
C. L. L. and Sube Singh, 2011, Genetic diversity for grain nutrients contents in a core collection of finger millet (Eleusine coracana (L.) Gaertn.) germplasm. Field Crops Res., 121: 4252.

Upadhyaya, H.D., Pundir, R.P.S. and Gowda, C.L.L., 2007. Genetic resources diversity of finger millet - a global perspective. Finger Millet blast management in East Africa. creating opportunities for improving production and utilization of finger millet. proceedings of the first international finger millet stakeholder workshop, projects R8030 and R8445 UK Department for international development - crop protection programme, 13-14 September 2005, Nairobi. International Crops Research Institute for the Semi-Arid Tropics, Patancheru 502324, Andhra Pradesh, India, pp. 90-101.

Vadivoo, A. S., Joseph, R. and Ganesan, N. M., 1998, Genetic diversity and variability for protein and calcium contents in finger millet (Elusine coracana L. Gaertn) in relation to grain colour. Plant Food Hum.Nutr., 52(9): 353-364.

\section{How to cite this article:}

Saritha, H.S., P. Ravishankar and Sunitha, N.C. 2018. Stability of Grain Nutrient Concentrations in White Finger Millet. Int.J.Curr.Microbiol.App.Sci. 7(11): 2786-2801. doi: https://doi.org/10.20546/ijcmas.2018.711.321 